\title{
Artículo Original \\ Prevalencia de Síndrome Metabólico en pacientes psoriáticos chilenos
}

\author{
Hernán Correa ${ }^{1,2}$, Daniela Jara ${ }^{2}$, Cristian Ramírez ${ }^{3}$, Paula Hasbún ${ }^{4}$, Ignacio Correa ${ }^{4}$, Paulina Cuevas ${ }^{4}$ y Rubén Orellana ${ }^{5}$
}

\section{RESUMEN}

Introducción: Existe poca información de la asociación entre Psoriasis y síndrome metabólico (SM) en Chile.

Objetivos: Determinar la prevalencia de SM en psoriáticos chilenos.

Material y métodos: Se realizó un estudio caso-control, con 487 pacientes (238 psoriáticos y 239 controles). En ambos grupos se analizó la prevalencia de SM además del exceso de peso, hipertensión arterial, diabetes mellitus 2, hipertrigliceridemia y niveles de HDL bajo.

Resultados: Los psoriáticos presentaron mayor probabilidad de padecer SM versus los controles $(35,29 \%$ vs $23,29 \%$ ) OR $1,8(1,20-2,66) \mathrm{p}<0,05$. Se asoció también con circunferencia abdominal aumentada $(57,14 \%$ vs 36,94$)$ OR 2,6 (1,79 - 3,78), exceso de peso $(85,99 \%$ vs $63,45 \%)$ OR $2,46(1,62-3,73)$ $\mathrm{p}<0,05$ y diabetes mellitus $2(13,45 \%$ vs $4,42 \%)$ OR $3,85(1,79$ - 8,26) $\mathrm{p}<0,05$.

Conclusiones: Se encontró una mayor prevalencia de SM, exceso de peso, circunferencia abdominal aumentada y diabetes mellitus 2 en el grupo psoriático en comparación al grupo control.

Palabras clave: Psoriasis; Síndrome metabólico; Diabetes Mellitus 2; Hipertensión; Hipertrigliceridemia; Exceso de peso; Circunferencia abdominal; Colesterol HDL.

\section{SUMmaRY}

Background: There is a paucity of information about Psoriasis and metabolic syndrome (MS) association in Chile.

Aim: To determine the MS prevalence in Chilean psoriatic patients.

Material and Methods: A case-control study was conducted which included 487 patients (238 psoriatic patients and 239 controls). In both groups, MS prevalence, overweight, hypertension, diabetes mellitus type 2, hypertriglyceridemia and low HDL were analyzed.

Results: Psoriatic patients were more likely to present MS than controls (35.29\% vs $23.29 \%$ ) OR 1.8 (1.20 to 2.66$) \mathrm{p}$ $<0.05$. In addition, it was associated with increased abdominal circumference $(57.14 \%$ vs 36.94$)$ OR 2.6 (1.79 to 3.78 ), overweight $(85.99 \%$ vs $63.45 \%$ ) OR 2.46 (1.62 to 3.73$) \mathrm{p}<0.05$ and diabetes mellitus type $2(13.45 \%$ vs. $4.42 \%)$ OR 3.85 (1.79 to 8.26$) \mathrm{p}<0.05$.

Conclusions: There is a higher prevalence of MS, overweight, increased abdominal circumference and type 2 diabetes mellitus in the psoriatic group compared with the control group.

Key words: Psoriasis; Metabolic Syndrome X; Type 2 Diabetes Mellitus; Hypertension; Hypertriglyceridemia; Overweight; Sagittal Abdominal Diameter; HDL Cholesterol.

\section{L} a psoriasis es una dermatosis inflamatoria crónica que afecta entre el 2 y $3 \%$ de la población general. ${ }^{1,2}$ Henseler y Christophers en 1995 analizan los datos de más de 40.000 pacientes psoriáticos y concluyen que existe de una mayor prevalencia de obesidad, diabetes mellitus e insuficiencia cardiaca en pacientes psoriaticos. $^{3}$

${ }^{1}$ Unidad docente asociada del Complejo Asistencial Dr. Sótero del Río. Escuela Medicina, Pontificia Universidad Católica de Chile, Santiago, Chile. ${ }^{2}$ Servicio Dermatología, Complejo Asistencial Dr. Sótero del Rio. Santiago, Chile. ${ }^{3}$ Médico Cirujano, Pontificia Universidad Católica de Chile, Santiago, Chile. ${ }^{4}$ Médico Cirujano, Universidad de Santiago, Santiago, Chile. ${ }^{5}$ Instituto de Sociología, Pontificia Universidad Católica de Chile. Santiago, Chile.

Correspondencia: Dr. Hernán Correa G. Correo electrónico: hcorreag@ uc.cl. Teléfono: +56 2 22335982. Dirección: Las Urbinas 81, departamento 2A. Providencia. Santiago. Código Postal: 7510093.
Desde entonces existe una preocupación creciente por establecer el vínculo preciso entre estas patologías metabólicas y la psoriasis.

El síndrome metabólico (SM) es una entidad diagnóstica cuya presencia se asocia a un mayor riesgo de diabetes y de enfermedades cardiovasculares en la población general. Se estima que afecta a cerca del $35,3 \%$ de la población chilena. ${ }^{4}$

El SM fue investigado en una revisión sistemática que incluye 12 publicaciones con casi 42.000 pacientes donde concluye que los pacientes psoriáticos presentan una prevalencia más alta de SM en comparación a la población general, especialmente en aquellos pacientes con enfermedad severa. ${ }^{5}$ Gisondi y cols. determinan en su estudio que el SM era más prevalente en pacientes con 
psoriasis que en controles pasados los 40 años de edad. Además, existe una mayor asociación de psoriasis con hipertrigliceridemia y obesidad abdominal que con el resto de los componentes del síndrome. ${ }^{6}$

En lo que se refiere a la asociación con hipertensión arterial (HTA), los estudios sugieren que el paciente psoriático tiene más riesgo de tener esta condición y a su vez la presencia de psoriasis hace más difícil el control de la hipertensión. ${ }^{7,8}$

La diabetes mellitus tipo 2 (DM 2) aparece con mayor frecuencia en pacientes psoriáticos cuando se compara con controles. ${ }^{7,9}$ En un metanálisis que incluye 27 estudios observacionales se concluye que la psoriasis tiene una prevalencia e incidencia elevada de diabetes y que esta asociación es más notoria en psoriasis severas. ${ }^{10}$ Aparentemente los pacientes psoriáticos serían más insulinoresistentes que los controles, por lo tanto, el riesgo de diabetes se vincula a defectos metabólicos propios de la psoriasis. ${ }^{11}$

Respecto de las hiperlipidemias, Neimann en su trabajo observa una prevalencia elevada en psoriasis moderada a severa. ${ }^{12}$

Dado que en Chile hay escasos estudios dirigidos a verificar $\mathrm{y} / \mathrm{o}$ cuantificar la relación entre la psoriasis y las comorbilidades mencionadas, creemos necesario investigar este tópico en la población local, ya que si se repiten los hallazgos mencionados en la literatura, podremos sugerir pautas de manejo de los pacientes psoriáticos que incluyan una evaluación de las comorbilidades detectadas y con ello mejorar no sólo el manejo de la patología cutánea, sino que eventualmente contribuir a mejorar la expectativa de vida de este grupo de pacientes.

Para esto diseñamos un estudio caso-control para determinar la prevalencia de SM en pacientes psoriáticos chilenos, establecer la frecuencia de DM2, HTA, exceso de peso, circunferencia abdominal aumentada, hipertrigliceridemia y niveles de HDL bajos en ambos grupos de estudio y analizar sus diferencias, además de establecer su relación con la severidad de psoriasis.

\section{Pacientes y métodos}

Para este estudio de casos y controles, los casos seleccionados son pacientes mayores de 18 años, con historia de lesiones cutáneas de al menos 6 meses de evolución diagnosticados de psoriasis por algún dermatólogo y que consultan al servicio de dermatología del Hospital Dr. Sótero del Río o centro dermatológico privado.

Los controles son pacientes mayores de 18 años, que consultan en el servicio de dermatología del Hospital Dr. Sótero del Río o centro dermatológico privado por enfermedades dermatológicas que no fuesen psoriasis. Además se incluyen pacientes de consulta de medicina general que solicitan un control de salud.

El periodo de selección de pacientes abarca desde noviembre 2012 hasta junio 2015.

Los pacientes excluidos del estudio son menores de 18 años, usuarios de tratamiento corticoidal crónico o agudo en los últimos siete días, insuficientes renales crónicos, pacientes con antecedentes de hipertensión secundaria y embarazadas.

Las variables a medir en ambos grupos son: peso $(\mathrm{Kg})$ y talla $(\mathrm{m})$, circunferencia abdominal $(\mathrm{cm})$, presión arterial $(\mathrm{mmHg})$, glicemia ayuno (mg/dL), HDL (mg/dL), triglicéridos $(\mathrm{mg} / \mathrm{dL})$ y prueba de tolerancia a glucosa oral (PTGO), esta última sólo se realiza a pacientes que cumpliesen criterios según lo establecido en la norma de Diabetes Mellitus MINSAL 2010. ${ }^{12}$ Las mediciones de circunferencia abdominal y de presión arterial se llevaron a cabo con una técnica estandarizada y un equipo calibrado. ${ }^{13,14}$ Con estas variables se determina la presencia o ausencia del SM según los criterios ATP III actualizados. ${ }^{15,16}$ Se consideran con la enfermedad los pacientes previamente diagnosticados o con tratamiento durante el último sin mediar nuevos exámenes de laboratorio.

En los pacientes psoriáticos además se evalúa la severidad de la psoriasis definida en leve, moderada y severa. Leve se determina con un compromiso $<10 \%$ de superficie corporal comprometida $(\mathrm{SCG})$, moderada con compromiso entre $10-19 \%$ de SCG, y severa con com- 
Tabla 1. Caracterización socio-demográfica de la población en estudio.

\begin{tabular}{llll}
\hline & Psoriáticos & Controles & Valor p \\
\hline $\mathrm{N}^{\circ}$ total de pacientes & 238 & 249 & NS \\
Edad = Promedio años \pm DE (rango) & $46,1 \pm 13,49$ & $45,2 \pm 15,89$ & $(18-82)$ \\
& $(18-90)$ & \\
Sexo & & 95 \\
$\mathrm{~N}^{\circ}$ Hombres & 139 & 154 \\
$\mathrm{~N}^{\circ}$ Mujeres & 99 & \\
\hline
\end{tabular}

$\overline{D E}=$ desviación estándar.

Tabla 2. Comorbilidades estudiadas en grupo psoriasis y grupo control.

\begin{tabular}{|c|c|c|c|c|}
\hline Comorbilidades (*) & $\begin{array}{l}\text { Psoriáticos } \\
\text { N }(\%)\end{array}$ & $\begin{array}{l}\text { Controles } \\
\text { N }(\%)\end{array}$ & $\begin{array}{c}\text { OR } \\
\text { (Intervalo de confianza) }\end{array}$ & Valor $\mathbf{P}$ \\
\hline $\mathrm{SM}$ & $84(35,29)$ & $58(23,29)$ & $1,8(1,2-2,69)$ & $<0,05$ \\
\hline $\mathrm{DM} 2$ & $32(13,45)$ & $11(4,42)$ & $3,85(1,79-8,26)$ & $<0,05$ \\
\hline HTA & $63(26,47)$ & $48(19,22)$ & $1,49(0,96-2,29)$ & NS \\
\hline Exceso de peso & $193(84,99)$ & $158(63,45)$ & $2,46(1,62-3,73)$ & $<0,05$ \\
\hline Circunferencia abdominal aumentada & $136(57,14)$ & $92(36,94)$ & $2,6(1,79-3,78)$ & $<0,05$ \\
\hline Hipertrigliceridemia & $37(15,54)$ & $33(13,25)$ & $1,2(0,73-2,00)$ & NS \\
\hline HDL bajo & $87(36,55)$ & $84(33,73)$ & $1,13(0,77-1,64)$ & NS \\
\hline
\end{tabular}

$N S=$ no significativo

(*)Se utilizan los siguientes criterios para cada patología

SM: Criterios ATPIII actualizado.

DM 2: Criterios diagnósticos guía Ministerio Nacional de Salud

HTA: Presión arterial sistólica y/o diastólica $\geq 140 / 90 \mathrm{mmHg}$.

Exceso de peso: $I M C \geq 25$

Circunferencia abdominal aumentada: $\geq 102 \mathrm{~cm}$ hombres $y \geq 88 \mathrm{~cm}$ mujeres.

Hipertrigliceridemia: Colesterol total $<200 \mathrm{mg} / \mathrm{dL}$. y triglicéridos $>150 \mathrm{mg} / \mathrm{dL}$.

HDL bajo: $\leq 40 \mathrm{mg} / \mathrm{dL}$. en hombres $y \leq 50 \mathrm{mg} / \mathrm{dL}$. en mujeres.

Tabla 3. Comparación de variables estudiadas entre psoriáticos y controles con SM.

\begin{tabular}{llll}
\hline Variables & $\begin{array}{l}\text { Psoriáticos con SM } \\
\text { (Promedio } \pm \text { DE) }\end{array}$ & $\begin{array}{l}\text { Controles con SM } \\
\text { (Promedio } \pm \text { DE) }\end{array}$ & Valor p \\
\hline $\mathrm{N}^{\circ}$ de pacientes & 84 & 58 & NS \\
IMC (Kg/m2) & $31,7 \pm 4.78$ & $31,1 \pm 4.65$ & $<0.04$ \\
Circunferencia abdominal (cm) & $108,3 \pm 11.63$ & $102,2 \pm 12.78$ & $<0.03$ \\
PA sistólica (mmHg) & $124,69 \pm 11,83$ & $118,81 \pm 19,02$ & $<0.001$ \\
PA diastólica (mmHg) & $83,11 \pm 10,04$ & $74,25 \pm 10,36$ & NS \\
Glicemia (mg/dL) & $100,35 \pm 29.62$ & $101,71 \pm 11.56$ & NS \\
Colesterol HDL en sangre (mg/dL) & $42,93 \pm 11.95$ & $42,76 \pm 9.77$ & NS \\
Triglicéridos en sangre (mg/dL) & $207,94 \pm 108.36$ & $207,16 \pm 132.3$ & \\
\hline
\end{tabular}

PA: Presión arterial

$D E$ : desviación estándar

NS: no significativo 
Tabla 4. Patologías metabólicas y su relación con la severidad de la psoriasis.

\begin{tabular}{lllll}
\hline Comorbilidades & $\begin{array}{l}\text { Psoriasis leve } \\
\mathbf{N}(\%)\end{array}$ & $\begin{array}{l}\text { Psoriasis Moderada } \\
\mathbf{N}(\%)\end{array}$ & $\begin{array}{l}\text { Psoriasis Severa } \\
\mathbf{N}(\%)\end{array}$ \\
\hline $\mathrm{N}^{\circ}$ de pacientes & 106 & 66 & 66 & Valor p \\
SM & $39(36,8)$ & $24(36,36)$ & $21(31,82)$ & NS \\
DM 2 & $18(16,98)$ & $7(10,61)$ & $8(12,13)$ & NS \\
HTA & $26(24,52)$ & $21(31,82)$ & $16(24,24)$ & NS \\
Exceso de peso & $86(81,13)$ & $55(83,3)$ & $52(78,79)$ & NS \\
Circunferencia abdominal aumentada & $63(59,43)$ & $33(50)$ & $40(60,61)$ & NS \\
Hipertrigliceridemia & $18(16,98)$ & $17(25,76)$ & $8(12,13)$ & NS \\
HDL Bajo & $37(34,90)$ & $28(37,38)$ & $22(33,33)$ & NS \\
\hline
\end{tabular}

NS: no significativo.

promiso $20 \%$ o más porcentaje de SCG. Para calcularla, se toma en consideración la palma del paciente, desde la muñeca a la punta de dedos, representando aproximadamente $1 \%$ de SGC.

Los datos se recolectaron en una planilla de recolección de datos diseñada para el estudio y se manejan de forma confidencial. Este estudio fue aprobado por el comité de ética del Hospital Dr. Sótero del Río - Servicio de Salud Metropolitano Sur Oriente.

El análisis estadístico es realizado mediante el programa SPSS versión 19 y STATA 12. Para evaluar la asociación de variables numéricas se utiliza la prueba t-Student y para variables dicotómicas se usa el test de $\chi 2$. Para evaluar la magnitud de la asociación entre variables se determina la razón de chance $(\mathrm{OR})$ y se considera significativo un valor $\mathrm{p}<0,05$.

\section{Resultados}

Participan un total de 487 pacientes, divididos en dos grupos: el primero conformado por 238 pacientes con el diagnóstico de psoriasis $(48,87 \%$ ) y el segundo por 249 pacientes que no tienen el diagnóstico de la enfermedad (51,13\%). Del grupo total participan 234 hombres y 253 mujeres. La caracterización socio-demográfica por grupos se presenta en la tabla 1.

La mayoría de las comorbilidades son más frecuentes en pacientes psoriáticos que en controles (Tabla 2). Además se evidencia que el 35,29\% de los psoriáticos presentaron SM mientras que sólo el 23,29\% de los controles tienen esta condición. Se observa la asociación entre psoriasis y
SM a través del test $\chi 2$ con un valor de $8,18(\mathrm{p}<0.05)$ y un OR de 1,8 (1,20 - 2,69).

El grupo con psoriasis se asocia a DM 2, exceso de peso y circunferencia abdominal aumentada. No hay asociación entre psoriáticos con HTA, hipertrigliceridemia, ni HDL bajo. (Tabla 2)

Dada la asociación entre SM y psoriasis, se comparan sólo los pacientes con SM de ambos grupos (psoriáticos y controles). La tabla 3 muestra que el grupo de psoriasis con SM presenta cifras elevadas en la mayoría de las variables estudiadas en comparación con el grupo control con SM, pero sólo es significativa la diferencia vista a nivel de circunferencia abdominal aumentada y de cifras tensionales $(\mathrm{p}<0.05)$

No hay asociación estadísticamente significativa entre severidad de psoriasis y las comorbilidades estudiadas (Tabla 4).

\section{Discusión}

Nuestro estudio concluye que existe un $80 \%$ más de riesgo de padecer SM en el grupo psoriasis en comparación al grupo control (Tabla 2). Sin embargo, al comparar con los datos de la encuesta nacional de salud 2009-2010 (ENS), vemos que el porcentaje de población chilena con SM, es muy similar a lo encontrado en el grupo psoriasis (35,3 vs 35,29\% respectivamente). ${ }^{4}$

Esto revela que nuestro grupo control presenta una menor prevalencia de SM que lo esperado en la población general, lo que los hace más saludables en este aspecto. Una posible explicación es que la ENS no recoja la situa- 
ción real de la población del país en este ítem. Por otro lado, puede ser que nuestros controles al ser usuarios de los servicios de salud sean pacientes atentos al estado de su salud, y por ende con estilos de vida más saludables que los no consultantes.

Con estos datos se hace difícil establecer una relación directa entre psoriasis y SM en la población chilena, a menos que se ignore la información de la ENS. Si es así, nuestros hallazgos están en línea con lo publicado en la literatura. ${ }^{5,6}$

En relación a exceso de peso, se concluye que hay 1,46 veces más chance de presentar IMC $\geq 25$ por los psoriáticos, lo que se acompaña de la alta prevalencia de exceso de peso en este grupo, con un 84,99\%. Esta cifra es superior al referente nacional de la ENS que registra un $64,5 \%$ y a lo encontrado en el grupo control.

Los controles en nuestro estudio con respecto al exceso de peso se comportan de forma muy similar a los datos de la ENS. ${ }^{4}$

Existen estudios donde se ve una mejoría de la psoriasis en pacientes que disminuyen su peso a través de dietas, ejercicio físico y cirugía bariátrica. ${ }^{17,18} \mathrm{y}$, se ha sugerido que la obesidad puede exacerbar la psoriasis. ${ }^{19} \mathrm{El}$ vínculo estaría dado por la acción de las citoquinas derivadas de adipocitos, en particular leptina y resistina que podrían estar involucradas en la patogénesis subyacente de ambas enfermedades, probablemente por un proceso mediado por aumento de la expresión de citoquinas proinflamatorias, principalmente IL-8, IL-12, IL-17 y TNFa. ${ }^{20}$ Se ha planteado la hipótesis que la leptina tendría un rol en la patogénesis de la enfermedad ${ }^{21}$, en tanto la resistina estaría relacionada con la severidad. ${ }^{22}$

La prevalencia de circunferencia abdominal aumentada en nuestro grupo de psoriáticos es de un $57,14 \%$ en tanto que los controles sólo presentan un 36,4\%. Estos resultados son más bajos en comparación a lo que publica Farias y cols, de 44,3\% en psoriáticos bajo los mismos criterios diagnósticos. ${ }^{23}$ Lamentablemente no podemos comparar con la ENS, ya que utilizan criterios más estrictos para el diagnóstico de circunferencia abdominal aumentada. ${ }^{4}$

La prevalencia de DM2 en psoriáticos es de un 13,45\%, lo que implica que tienen 2,85 veces más riesgo de padecer DM2 en comparación al grupo control. La ENS publica un $9,4 \%$ de prevalencia nacional de DM2, cifra que es mayor a nuestra prevalencia de 4,42\% del grupo control. ${ }^{4}$ Una posible explicación a esta discordancia de los controles con la ENS es el hecho de que los pacientes con DM2, son canalizados mayoritariamente a la consulta de morbilidad y no a controles de salud preventivos.

Sobre la prevalencia de HTA, nuestro grupo de psoriasis tiene cifras similares al referente nacional $(26,47 \%$ vs $26,9 \%$ respectivamente), ${ }^{4}$ sin embargo en comparación a nuestro grupo control no existe una asociación significativa con psoriasis. (Tabla 2)

Ma y cols. resportan en su trabajo que existe asociación entre psoriasis y dislipidemia, definida bajo distintos criterios lo que hace difícil su comparación con nuestro estudio. ${ }^{24}$ Aun así, esto se contrapone a lo visto en nuestro trabajo donde no se encuentra asociación entre psoriasis y HDL bajo, ni tampoco hipertrigliceridemia.

Dentro del grupo de pacientes con SM, se evidencia que los psoriáticos tienen mayor circunferencia abdominal y mayores cifras tensionales que el grupo control (Tabla 3). Esto permite inferir que el ser psoriático aportaría un riesgo cardiovascular independiente, hecho que se debe confirmar con estudios prospectivos.

Armstrong y cols, concluyen en su revisión sistemática que existe una asociación entre la severidad de la psoriasis y la presencia de $\mathrm{SM},{ }^{5}$ hecho que en nuestro estudio no es corroborado (Tabla 4).

Se piensa que el síndrome metabólico se origina en la resistencia a la insulina y la función anormal del adipocito. ${ }^{25}$ Como ya se ha mencionado, la literatura es pródiga en datos que le atribuyen una mayor prevalencia en los pacientes con psoriasis que en la población general. Hay varios mecanismos biológicos que podrían explicar esta relación. En primer lugar, podemos mencionar que la elevación crónica de los ácidos grasos libres lleva a una disfunción del adipocito, condición que se observa tanto en el síndrome metabólico como en la psoriasis, lo que determina una inhibición de la secreción de insulina conduciendo a una diabetes tipo $2 .{ }^{26}$

Otro hecho significativo es que algunos locus de riesgo genético compartido como CDKAL1 están asociados a psoriasis y diabetes tipo $2,{ }^{27}$ en tanto que el PTPN22 está asociado con psoriasis y diabetes tipo $1 .^{28}$

Por último, el desbalance en la función de las adipocitoquinas en psoriasis también contribuye a explicar su relación con el síndrome metabólico. Un rol destacado 
corresponde a la leptina que es una citoquina proinflamatoria que se ha reportado se encuentra en niveles más altos en pacientes con psoriasis. ${ }^{22} \mathrm{Su}$ importancia radica en que se le asigna un rol significativo en le enfermedad arteriosclerótica.$^{29}$ Por otro lado el déficit de adiponectina, que tiene un rol protector, también ha sido descrito con bastante frecuencia. ${ }^{30}$

Es a partir de estos factores mencionados que se ha establecido que en la psoriasis, al igual que en otras enfermedades crónicas inflamatorias, se dan las condiciones para que se asocie al síndrome metabólico o alguno de sus componentes, todos los cuales tendrán influencia en la salud general del paciente, principalmente la enfermedad cardiovascular. Así entonces, se entiende la preocupación creciente por las comorbilidades que acompañan al paciente psoriático y la importancia de su diagnóstico precoz y manejo adecuado con el fin de mejorar no sólo la calidad de vida del paciente sino que, además, su expectativa de vida.

\section{Conclusiones}

Los resultados de nuestra investigación sugieren que los pacientes con psoriasis tienen mayor prevalencia de exceso de peso, circunferencia abdominal aumentada y DM2 que aumentan su riesgo cardiovascular. Sin embargo, la prevalencia de SM y su asociación con psoriasis, no quedan tan clara al comparar los datos con la ENS. Aun cuando no hemos podido reproducir los resultados que se encuentran en la mayor parte de la literatura médica sobre el tema, creemos que estos hallazgos son importantes para la práctica clínica en el medio local, ya que nos acercan a la realidad epidemiológica de nuestros pacientes. Ello nos permitirá un fundamento valioso para ofrecer un enfoque integral a estos pacientes, identificar a los que presentan un mayor riesgo cardiovascular e iniciar el tratamiento precoz de las enfermedades asociadas.

Dada la diferencia con los hallazgos reportados en la literatura, creemos que se debe insistir en futuros estudios sobre el tema que ayuden a conocer con exactitud la realidad local lo que nos permitirá un manejo más adecuado de nuestros pacientes.

\section{REFERENCIAS BIBLIOGRÁFICAS}

1. Sánchez L. Aspectos Epidemiológicos en Psoriasis. En: Sánchez L. Psoriasis. $1^{a}$ Edición, Santiago: Actualidades Médicas, 1999; 13-20

2. Neimann AL, Porter S, Gelfand JM. Epidemiology of psoriasis. Expert Rev Dermatol 2006; 1: 63-75

3. Henseler T, Christophers E. Disease concomitance in psoriasis. J Am Acad Dermatol 1995; 32(6):982-986

4. Ministerio de salud, Universidad Católica de Chile, Universidad Alberto Hurtado. Encuesta nacional de salud, Chile 2009- 2010. Santiago. Minsal 2010. Disponible en: http://web.minsal.cl/portal/ url/item/bcb03d7bc28b64dfe040010165012d23.pdf. [Consultado el 19 de mayo de 2016]

5. Armstrong AW, Harskamp CT, Armstrong EJ. Psoriasis and metabolic syndrome: a systematic review and meta-analysis of observational studies. J Am Acad Dermatol 2013; 68(4): 654-662

6. Gisondi P, Tessari G, Conti A, Piaserico S, Schianchi S, Peserico A, et al. Prevalence of metabolic syndrome in patients with psoriasis: a hospital based case-control study. Br J Dermatol 2007; 157(1):68-73

7. Qureshi AA, Choi HK, Setty AR, Curhan GC. Psoriasis and the risk of diabetes and hypertension: a prospective study of US female nurses. Arch Dermatol 2009; 145(4):379-382

8. Takeshita J, Wang S, Shin DB, Mehta NN, Kimmel SE, Margolis DJ, et al. Effect of psoriasis severity on hypertension control: a population-based study in the United Kingdom. JAMA Dermatol 2015; 151(2):161-169

9. Neimann AL, Shin DB, Wang X, Margolis DJ, Troxel AB, Gelfand JM. Prevalence of cardiovascular risk factors in patients with psoriasis. J Am Acad Dermatol 2006; 55(5):829-835

10. Armstrong AW, Harskamp CT, Armstrong EJ. Psoriasis and the risk of diabetes mellitus: a systematic review and meta-analysis. JAMA Dermatol 2013; 149(1):84-91

11. Gyldenløve M, Storgaard H, Holst JJ, Vilsbøll T, Knop FK, Skov L. Patients with psoriasis are insulin resistant. J Am Acad Dermatol 2015; 72(4):599-605

12. Ministerio de Salud. Guia Clínica Diabetes Mellitus tipo 2.Santiago, Minsal 2010. Disponible en: www.minsal.cl [Consultado el 19 octubre 2012]

13. Moreno M. Circunferencia de cintura: una medición importante y útil del riesgo cardiometabólico. Rev Chil Cardiol 2010; 29:85-87

14. Ministerio de Salud. Guía Clínica Hipertensión Arterial Primaria o esencial en personas de más de 15 años. Santiago, Minsal 2010. Disponible en: www.minsal.cl [Consultado el 19 octubre 2012]

15. Expert Panel on Detection, Evaluation, and Treatment of High Blood Cholesterol in Adults. Executive Summary of The Third Report of The National Cholesterol Education Program (NCEP) Expert Panel on Detection, Evaluation, And Treatment of High Blood Cholesterol In Adults (Adult Treatment Panel III). JAMA 2001; 285(19):2486-2497

16. Grundy SM, Brewer HB Jr, Cleeman JI, Smith SG Jr, Lenfant C; American Heart Association; National Heart, Lung, and Blood Institute. Definition of metabolic syndrome: Report of the National Heart, Lung, and Blood Institute/American Heart Association conference on scientific issues related to definition. Circulation 2004; 109(3):433-438 
17. Naldi L, Conti A, Cazzaniga S, Patrizi A, Pazzaglia M, Lanzoni A, et al. Diet and physical exercise in psoriasis: a randomized controlled trial. BrJ Dermatol 2014 Mar; 170(3):634-42

18. Romero-Talamás H, Aminian A, Corcelles R, Fernandez AP, Schauer PR, Brethauer S. Psoriasis improvement after bariatric surgery. Surg Obes Relat Dis 2014; 10(6):1155-1159

19. Sterry W, Strober BE, Menter A. Obesity in psoriasis: the metabolic, clinical and therapeutic implications. Report of an interdisciplinary conference and review. Br J Dermatol 2007; 157:649-55

20. Meijer K, de Vries M, Al-Lahham S et al. Human primary adipocytes exhibit immune cell function: adipocytes prime inflammation independent of macrophages. PLoS One 2011; 6:e17154

21. Hamminga EA, van der Lely AJ, Neumann HA, Thio HB. Chronic inflammation in psoriasis and obesity: implication for therapy. Med Hypotheses 2006; 67:768-73

22. Pina T, Genre F, Lopez-Mejias R. JEADV 2015, 29, 1995-2001. Relationship of Leptin with adiposity and inflammation and Resistin with disease severity in Psoriatic patients undergoing anti-TNF-alpha therapy

23. Farias M, De la Cruz C, Romero W, Serrano V, Castillo V, Rozowski N. Estudio transversal: Evaluación de prevalencia de comorbilidades metabólicas y factores de riesgo cardiovascular, y su correlación con la severidad clínica, en 106 pacientes con psoriasis. Rev Chilena Dermatol 2012; 29(2):138-145
24. Ma C, Harskamp CT, Armstrong EJ, Armstrong AW. The association between psoriasis and dyslipidaemia: a systematic review. BrJ Dermatol 2013; 168(3):486-495

25. Olufadi R, Byrne CD. Clinical and laboratory diagnosis of themetabolic syndrome. J Clin Pathol 2008; 61:697-706

26. Zhao YF, Feng DD, Chen C. Contribution of adipocyte-derived factors to beta-cell dysfunction in diabetes. Int J Biochem Cell Biol 2006; 38:804-19

27. Wolf N, Quaranta M, Prescott NJ, Allen M, Smith R, Burden AD, et al. Psoriasis is associated with pleiotropic susceptibility loci identified in type II diabetes and Crohn disease. J Med Genet 2008; 45:1 14-6

28. Chen YF, Chang JS. PTPN22 C1858T and the risk of psoriasis: a meta-analysis. Mol Biol Rep 2012; 39:7861-70. Epub 2012 Apr 28

29. Karaduman M, Oktenli C, Musabak U et al. Leptin, soluble interleukin-6 receptor, C-reactive protein and soluble vascular cell adhesion molecule-1levels in human coronary atherosclerotic plaque. Clin Exp Immunol 2006; 143:452-457

30. Shibata S, Saeki H, Tada Y, Karakawa M, Komine M, Tamaki K. Serum high molecular weight adiponectin levels are decreased in psoriasis patients. J Dermatol Sci 2009; 55:62-3 